Received: 2014.07 .08 Accepted: 2014.07.22 Published: 2014.11.12

\title{
Simultaneous Pancreas and Kidney Composite Graft Transplantation with Retroperitoneal Systemic-Enteric Drainage
}

Authors' Contribution: Study Design A Data Collection B Statistical Analysis C Data Interpretation D Manuscript Preparation E Literature Search F Funds Collection $G$
ABCDEF 1,2 Shinichiro Ono

EF 2 Tamotsu Kuroki

F 2 Amane Kitazato

F 2 Tomohiko Adachi

EF 1 Yin-Yin Chen

F 1 Shin-Chin Chen

EF 2 Susumu Eguchi

ABCDEF 1 Yi-Ming Shyr

ABCDEF 1 Shin-E Wang
1 Departments of Surgery, Taipei Veterans General Hospital, National Yang Ming University, Taipei, Taiwan

2 Department of Surgery, Nagasaki University, Graduate School of Biomedical Sciences, Nagasaki, Japan
Corresponding Author: Source of support:

Background: Simultaneous pancreas and kidney transplantation is the treatment of choice for diabetes mellitus patients with associated end-stage renal disease. Limited vascular access could be encountered in patients with severe atherosclerosis, and/or severe obesity and in re-transplant patients. We describe a modified technique that facilitates simultaneous pancreas and kidney (SPK) composite graft transplantation with retroperitoneal systemic-enteric drainage for patients with limited vascular access.

Material/Methods: Since April 2012, we performed a modified technique for 2 patients with limited vascular access. SPK composite graft was constructed during the back-table preparation and transplanted in the right retroperitoneal space, finally covered by the ascending colon and its mesocolon.

Results: The 2 patients achieved good pancreas grafts function with normal blood glucose immediately after the completion of reperfusion. Their kidney grafts have also shown good function. They have not had any rejection episodes or postoperative complications after the SPK composite graft transplantation.

Conclusions: We propose that simultaneous pancreas and kidney composite graft transplantation with retroperitoneal systemic-enteric drainage can be a viable option for patients with limited vascular access.

MeSH Keywords: Kidney Transplantation - Pancreas Transplantation • Retroperitoneal Space

Abbreviations: $\quad \mathbf{C T}$ - computed tomography; DM - diabetes mellitus; IVC - inferior vena cava; SPK - simultaneous pancreas and kidney

Full-text PDF:

1790 腿尚 2 世尚 3 非当 26




\section{Background}

SPK is an established treatment option for brittle diabetes with associated end-stage renal disease. The success rate of pancreas transplantation has improved dramatically with advances in surgical techniques [1,2], graft preservation [3,4], novel immunosuppression $[1,5,6]$ and post-transplant management $[1,5]$. It is well known that microangiopathy and macroangiopathy are generalized with high probability in diabetes mellitus (DM) patients [7], and that these angiopathies can lead to advanced atherosclerotic arterial diseases whichthat may present technical problems for vascular anastomosis during graft implantation. Possible solutions for such severe atherosclerosis include patch reconstruction using a donor iliac artery [8] and arterial replacement using a donor iliac artery for interposition or bypass $[9,10]$.

However, patients with advanced atherosclerotic arterial disease, patients who are obese, and re-transplant patients may have limited access for 2 vascular anastomoses in multi-organ transplantation such as SPK. Reports had shown that when vascular access is limited, back-table reconstructions of combining pancreas and kidney grafts into a single artery and single vein or two veins may provide a successful outcome [11-13].

Most centers use enteric drainage and an intraperitoneal position as the standard technique for pancreas transplantation. Boggi first described an intraperitoneal approach for retroperitoneal pancreas graft placement with portal-enteric drainage [14]. A modified technique using systemic-enteric drainage was reported a few years later [15].

Here, we describe modifications that facilitated SPK composite graft transplantation with retroperitoneal systemic-enteric drainage for patients with limited vascular access and renal failure under hemodialysis.

\section{Material and Methods}

Since April 2012, we performed 2 SPK composite graft transplantation with retroperitoneal systemic-enteric drainage.The characteristics of the 2 recipients and the donors are shown in Table 1.

\section{Surgical technique}

\section{Organ procurement}

The pancreas was recovered with a duodenal segment without spleen. The spleen had already been removed during the organ procurement. The portal vein was divided near the confluence of the splenic vein to obtain a $1.5-2.0 \mathrm{~cm}$ length. During
Table 1. Recipient and donor demographics.

\begin{tabular}{|ccc}
\hline & Case 1 & Case 2 \\
\hline Recipient age (years) & 28 & 52 \\
\hline Gender & Male & Male \\
\hline BMI $\left(\mathrm{kg} / \mathrm{m}^{2}\right.$ ) & 33 & 23 \\
\hline Type of diabetes & NIDDM & IDDM \\
\hline Duration of diabetes (years) & 12 & 29 \\
\hline Duration of hemodialysis (years) & 2 & 4.5 \\
\hline Pannel reactive antibodies (\%) & 0 & 0 \\
\hline Donor age (years) & 57 & 40 \\
\hline HLA-mismatch & 0 & 3 \\
\hline Cold ischemia time (minutes) & 385 & 555 \\
\hline Warm ischemia time (minutes) & 47 & 49 \\
\hline
\end{tabular}

the back-table preparation, all vascular anastomosis were performed by 7-0 Prolene running sutures. The superior mesenteric artery and the splenic artery of the pancreas graft were reconstructed with a long $Y$ iliac artery graft. The smaller left renal artery was anastomosed end-to-side to the larger and longer common limb of the arterial $Y$ graft from the donor. The shorter portal vein is anastomosed end-to-side to the longer graft left renal vein (Figure 1). This composite graft facilitated the SPK transplantation using a single graft artery and vein for the anastomosis to 1 pair of recipient arterial and venous sites.

\section{Transplantation}

Through a midline incision, the bowel was mobilized from the cecum to the region of the hepatic flexure and mesocolon. The range of the mesocolon mobilization was expanded to expose the distal aorta, including bifurcation of common iliac arteries. After the mobilization was completed, a preventive appendectomy was performed in both cases due to potential difficulty in distinguishing graft complications from appendicitis in case of right inferior abdominal pain. The distal aorta, right common iliac, and right external and right internal arteries were encircled and the most suitable site for anastomosis was carefully determined. The atherosclerosis and obesity was so severe in these 2 patients that after extensive exploration of the vessels, it was still difficult to identify 2 segments of artery without calcification or 2 good vascular access points for arterial anastomosis. The severe atherosclerosis was later confirmed by post-operative CT image (Figure 2). By these findings, we decided to convert from conventional SPK to en block SPK composite graft transplantation. Next, dissection around the inferior vena cava (IVC), right common iliac vein, 


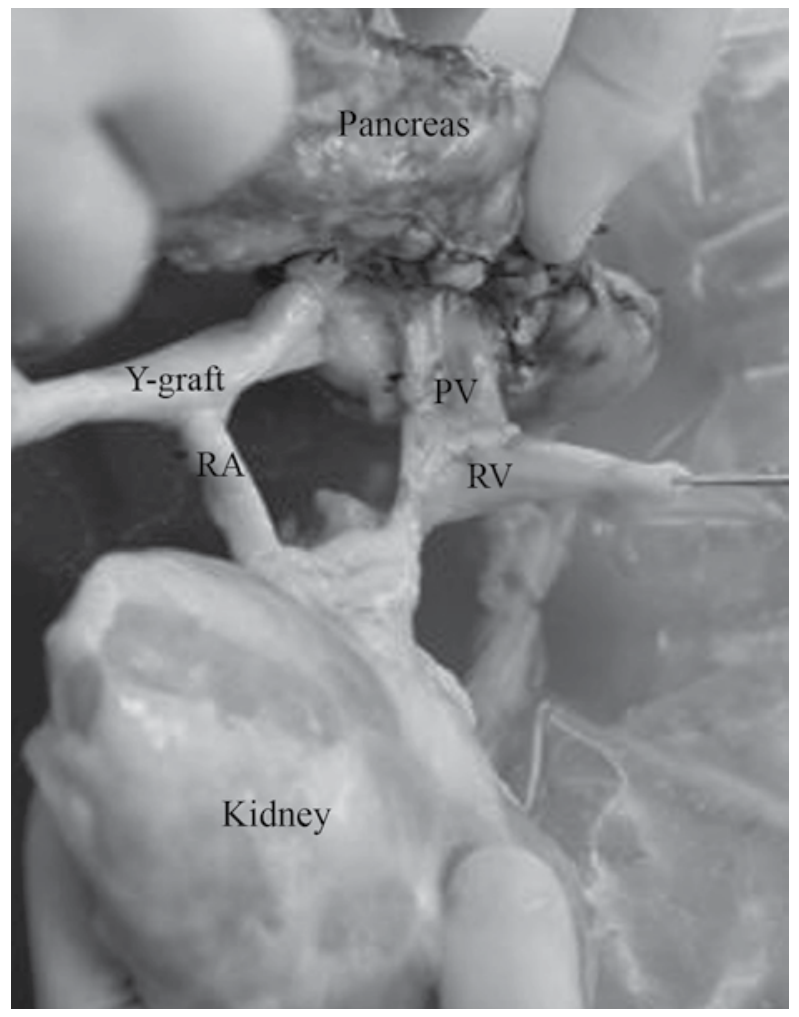

Figure 1. The smaller left renal artery was anastomosed endto-side to the larger and longer common limb of the arterial $Y$ graft and the shorter portal vein was anastomosed end-to-side to the longer graft left renal vein. PV - portal vein, RA - renal artery, RV - renal vein.

and right external iliac vein was performed until the anterior and lateral walls of these veins were exposed. After the vascular preparation was finished, a roux-en-Y jejunal limb was constructed ( $>40 \mathrm{~cm}$ long). The most important point was to avoid any tension of the mesentery. The pancreas and kidney composite graft renal vein was anastomosed to the IVC or the common iliac vein, and graft common iliac artery was anastomosed to the recipient distal aorta or the right external iliac artery using 6-0 Prolene running sutures. By bringing a jejunal limb through a hole in an avascular part of the right mesocolon, exocrine drainage was provided by anastomosis of the graft duodenum to a roux-en-Y jejunal limb in a side-to-side fashion by 2 -layer sutures. The ureter was anastomosed to the urinary bladder using a standard technique of extravesicular ureteroneocystostomy. Finally, closed drains were placed around the pancreas and the kidney composite graft, and then the colon and mesocolon were returned to their initial positions, allocating the composite graft in the retroperitoneum (Figure 3).

\section{Immunosuppression}

The induction therapy consisted of intraoperative basiliximab (20 mg) for induction and subsequent doses on Day 4.

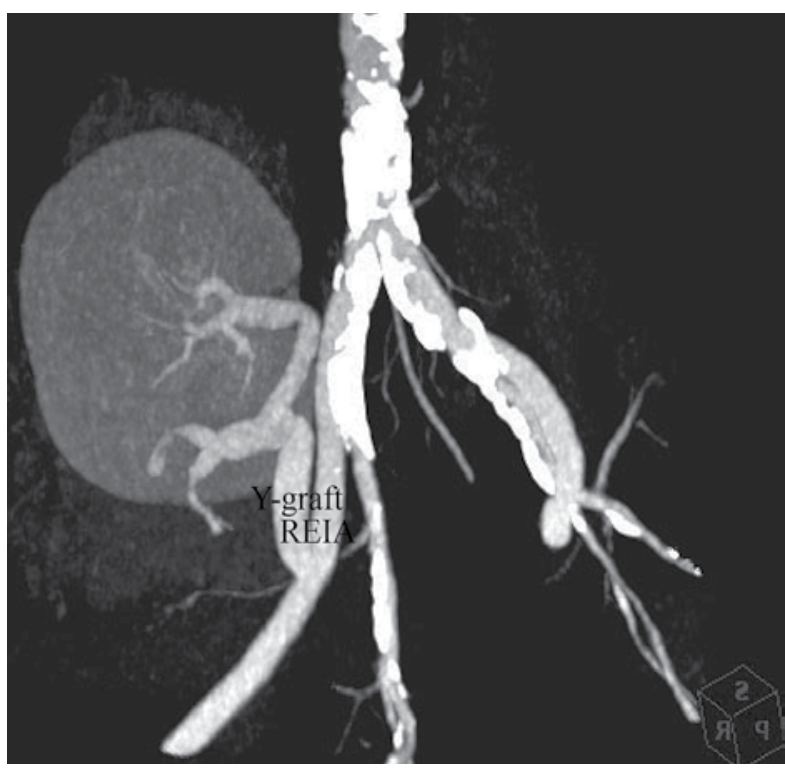

Figure 2. Severe atherosclerosis was noted from distal aorta to bilateral iliac arteries, except right external iliac artery. SPK composite was transplanted to right external iliac artery. REIA - right external iliac artery.

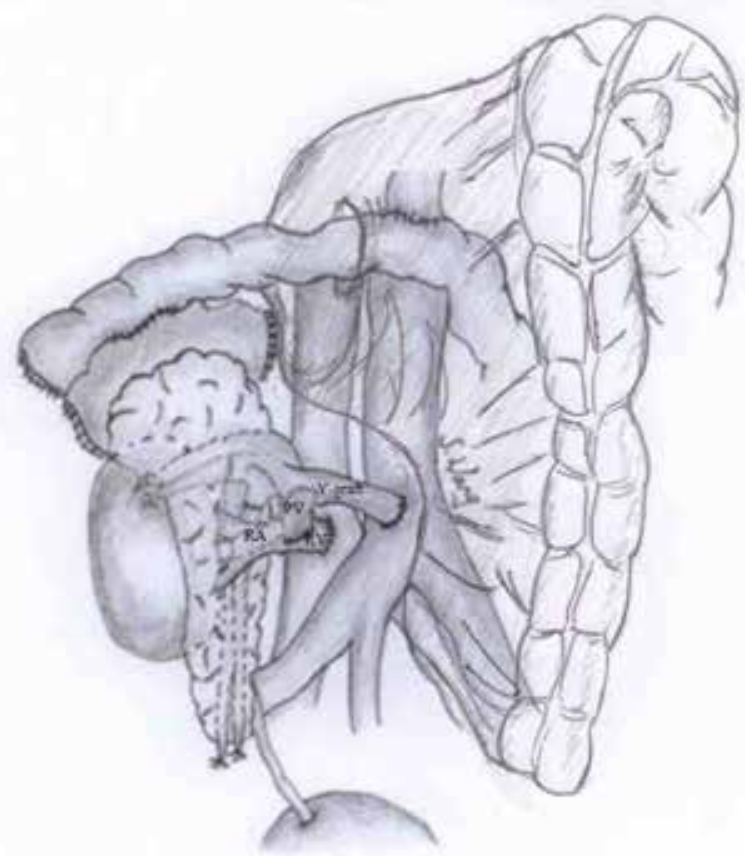

Figure 3. Simultaneous pancreas and kidney composite graft transplantation with retroperitoneal systemic-enteric drainage. A SPK composite graft's left renal vein was anastomosed to the recipient IVC and the Y-graft's common iliac artery was anastomosed to the recipient distal aorta. Bringing a jejunal limb through a hole in an avascular part of right mesocolon, exocrine drainage was provided by anastomosis of the graft duodenum to a roux-en-Y jejunal limb in a side-to-side fashion. PV - portal vein, RA - renal artery, RV - renal vein. 
Tacrolimus (0.05 mg/kg: target trough level was 6-15 ng/ml) and mycophenolate mofetil (1000 mg) were started twice daily for maintenance immunosuppression. Prednisolone was also used for maintenance, then tapered and withdrawn completely by 3 months after the transplantation.

\section{Perioperative management}

Ciprofloxacin or third-generation cephalosporin, and ampicillin were used for prophylaxis of surgical site infection for 7 days. Cytomegalovirus prophylaxis was routinely performed with intravenous ganciclovir for 7 postoperative days, followed by oral valganciclovir for 100 days, with dose adjustments for renal functions. Fungal prophylaxis was carried out by micafungin sodium (50 mg) for 7 days, followed oral nystatin $(10 \mathrm{~mL}$, 4 times daily) for 6 months.

For antithrombotic prophylaxis, we used oral aspirin (100 mg/day). In our criteria, we do not use calcium heparin, low-molecular weight heparin, or warfarin for antithrombotic prophylaxis.

When the drainage amount and amylase level from the drains were low, and there was no sign of infection or leakage, the closed drains were immediately removed.

\section{Results}

All grafts showed good function with normal blood glucose immediately at completion of reperfusion. The 2 patients' operative times were $7 \mathrm{~h}$ and $10 \mathrm{~h}$. The cold ischemia times were 6.4 $\mathrm{h}$ and $9.3 \mathrm{~h}$, and the warm ischemia times were $47 \mathrm{~min}$ and 49 $\min$. The patients were discharged on postoperative days 20 and 22. Three months after transplantation, pancreas and kidney of both of the patients showed good function. The hemoglobin A1C values were $5.9 \%$ and $5.2 \%$ at 3 months after the operation. Their serum creatinine levels were $1.6 \mathrm{mg} / \mathrm{dL}$ and $1.54 \mathrm{mg} / \mathrm{dL}$ (Table 2). They have had no rejection episodes or postoperative complications after the SPK composite graft transplantation.

\section{Discussion}

Candidates for pancreas transplantation often have complications such as neuropathy, retinopathy, and nephropathy, and are also very likely to have some degree of microangiopathy and macroangiopathy [7]. It is not uncommon to see severe atherosclorosis, which is often the most important factor in the morbidity and mortality of patients with type $1 \mathrm{DM}$ $[16,17]$. Atherosclerosis is a leading cause of limited vascular access and is an obstacle in achieving arterial anastomosis. One of the solutions to the limited vascular access problem is a SPK composite graft transplantation with single conduit of
Table 2. Postoperative course.

\begin{tabular}{ccc}
\hline & Case 1 & Case 2 \\
\hline Hospital stay (days) & 20 & 22 \\
\hline Complications & None & None \\
\hline HbA1C: 3 months (\%) & 5.9 & 5.2 \\
\hline Serum creatinine: 3 months $(\mathrm{mg} / \mathrm{dl})$ & 1.6 & 1.54 \\
\hline
\end{tabular}

arterial inflow and venous outflow by back-table preparation $[11,12]$. This concept was first described by Sugitani et al. [11], but their technique required portal vein elongation and double lumen venous drainages. Sasaki et al. later reported another techinique that differs from Sugitani's regarding the point of venous reconstruction. Sasaki et al. anastomosed the portal vein to the renal vein as a single conduit without portal vein elongation. In our 2 patients, we performed the similar vascular reconstruction using Sasaki's technique for patients with a limited vascular access point due to atherosclerosis and a limited transplantation space due to obesity. In our opinion, the advantages of this technique are that the reconstruction of the composite graft can be facilitated easily ex vivo and vascular anastomoses access is good both ex vivo and in vivo. In addition, the warm ischemic time can be reduced because only 1 pair of vascular anastomoses is needed for both the pancreas and kidney grafts. The disadvantage of this technique is that any complication (especially vascular thrombosis) that occurs in 1 graft might jeopardize the other graft. This technique must therefore be used carefully. Sasaki stated that the use of this technique can minimize vessel dissection; however, we think that prolonged exploration of vessels for suitable vascular anastomosis site is needed when dealing with such a delicate technique.

Since the first successful simultaneous pancreas and kidney transplantation was performed in 1966 by Kelly et al., from the University of Minnesota [18], many ideal surgical techniques have been suggested. In recent years, in most of the institutions that provide pancreas transplantation, the pancreas was transplanted on the right side and the kidney graft was transplanted on the left side with the arterial and venous anastomosis to bilateral common iliac artery and vein. With regard to exocrine drainage, ever since Nghiem and Corry described a modified bladder drainage technique [19], bladder drainage has been the standard. The introduction of calcineurin inhibitors improved the results of pancreas transplantations, and pancreas transplantation surgeons have wanted to reevaluate the role of enteric drainage for overcoming the poor quality of life caused by urinary tract complications related to nonphysiological drainage of pancreatic enzymes into the bladder $[20,21]$. Since the safety of enteric drainage compared to bladder drainage was reported [22-25], enteric 
drainage has been the standard technique at most pancreas transplantation centers. In 2004, Fridell et al. reported ipsilateral placement of pancreas and kidney transplantation [26]. Boggi et al. described intraperitoneal approach of ipsilateral retroperitoneal pancreas transplantation with portal-enteric drainage in 2005 [14], and modification of the Boggis technique using systemic venous drainage was later reported by Kahn et al. [15]. The retroperitoneal pancreas transplantation technique requires only mobilization of the right colon and mesocolon, and thus is not difficult. However, it has several advantages compared to conventional intraperitoneal pancreas transplantation; for example, easy access and dissection of the vascular anastomosis site, and easy reconstruction of arterial anastomosis, especially with severe obesity and atherosclerosis. The technique also provides post-transplant advantages, such as decreased risk of intestinal obstruction by separation of small bowels from the pancreas graft, reduced incidence of fluid collection, and the elimination of the spreading of an intra-abdominal infection if it occurs. In addition, the

\section{References:}

1. Sollinger HW, Odorico JS, Becker YT et al: One thousand simultaneous pancreas-kidney transplants at a single center with 22-year follow up. Ann Surg 2009; 250: 618-30

2. Boggi U, Vistoli F, Chiaro MD et al: Surgical techniques for pancreas transplantation. Curr Opin Organ Transplant, 2005; 10: 155-68

3. Fridell JA, Agarwal A, Milgrom ML et al: Comparison of histidine-tryptophanketoglutarate solution and University of Wisconsin perfusion solution in clinical pancreas transplantation. Transplantation, 2004; 77: 1304-6

4. Fridell JA, Mangus RS, Powelson JA: Histidine-tryptophan-ketoglutarate for pancreas allograft preservation: The Indiana university experience. Am J Transplant, 2010; 10: 1284-89

5. Sutherland DER, Gruessner RWG, Dunn DL et al: Lessons learned from more than 1,000 pancreas transplants at a single institution. Ann Surg, 2001; 233: 463-501

6. Kaufman DB, Leventhal JR, Gallon LG et al: Technical and immunologic prog ress in simultaneous pancreas-kidney transplantation. Surgery, 2002; 132 545-53

7. Mazzone T: Current concepts and controversies in the pathogenesis, prevention, and treatment of the macrovascular complications of diabetes. J Lab Clin Med, 2000; 135: 437-43

8. Mercer DF, Rigley T, Stevens RB: Extended donor iliac arterial patch for vascular reconstruction during pancreas transplantation. Am J Transplant, 2004; 4: 834-37

9. Moon J, Ciancio G, Burke GW: Arterial reconstruction with donor iliac vessels during pancreas transplantation: An intraoperative approach to arterial injury or inadequate flow. Clin Transplant, 2005; 19: 286-90

10. Fridell JA, Gage E, Goggins WC, Powelson JA: Complex arterial reconstruction for pancreas transplantation in recipients with advanced arteriosclerosis. Transplantation, 2007; 83: 1385-88

11. Sugitani A, Gritsh HA, Egidi F et al: En block pancreas kidney transplantation in a patient with limited vascular access. Transplantation, 1997; 63 1683-85

12. Sasaki TM, Light JA: Single unit simultaneous pancreas kidney graft facilitates transplantation. Transplantation, 1999; 68: 1432

13. Tso PL, Cash MP, Pearson TC et al: Simultaneous pancreas-kidney transplantation utilizing a common arterial conduit: early experience and potential applications. Am J Transplant, 2003; 3: 1440-43 retroperitoneal position of the graft provides good accessibility for a percutaneous biopsy to monitor rejection.

\section{Conclusions}

We have used the intraperitoneal approach for retroperitoneal pancreas graft placement with systemic-enteric drainage since 2008 , and in this report we describe the combination of this technique and the SPK composite graft technique for patients with limited vascular access due to severe atherosclerosis and obesity. Both of our patients have progressed favorably, with good function of both pancreas and kidney grafts after transplantation. In patients with limited vascular access, this method might be a good option for all pancreas transplantation surgeons.

\section{Conflict of interest statement}

Shinichiro Ono and other co-authors have no conflict of interest.

14. Boggi U, Vistoli F, Signori S et al: A technique for retroperitoneal pancreas transplantation with portal-enteric drainage. Transplantation, 2005; 79: 1137-42

15. Kahn J, Iberer F, Kniepeiss D et al: Retroperitoneal pancreas transplantation with systemic-enteric deainage - case report. Clin Transplant, 2008 22: 674-76

16. Pyoraia K, Laakso M, Unsitupa M: Diabetes and atherosclerosis: an epidemiologic vies. Diabetes Metab Rev, 1987; 3: 463-524

17. Larsen JL, Lynch T, Al'Halawani $M$ et al: Carotid intima-media thickness by ultrasound measurement in pancreas transplant candidates. Transplant Proc, 1995; 27: 2996

18. Kelly WD, Lillehei RC, Merkel FK et al: Allotransplantation of the pancreas and duodenum along with the kidney in diabetic nephropathy. Surgery, 1967; 61: 827-37

19. Nghiem DD, Corry RJ: Transplantation with urinary drainage of pancreatic secretions. Am J Surg, 1987; 153: 405-6

20. Mühlbacher F, Guant MF, Auinger $M$ et al: Pancreatic venous drainage to the portal vein: a new method in pancreas transplantation. Transplant Proc, 1990; 22: 636-37

21. Shokouh-Amiri MH, Gaber AO, Gaber LW et al: Pancreas transplantation with portal venous drainage and enteric exocrine diversion: a new technique. Transplant Proc, 1992; 24: 776-77

22. Gaber AO, Shohouh-Amiri MH, HathawayDK et al: Results of pancreas transplantation with portal venous and enteric drainage. Ann Surg, 1995; 221: 613-24

23. Bloom RD, Olivares $M$, Rehman L et al: Longterm pancreas allograft outcome in simultaneous pancreas-kidney transplantation: a comparison of enteric and bladder drainage. Transplantation, 1997; 64: 1689-95

24. Kuo PC, Johnson LB, Schweitzer EJ, Bartlett ST: Simultaneous pancreas kidney transplantation - a comparison of enteric and bladder drainage of exocrine pancreatic secretions. Transplantation, 1997; 63: 238-43

25. Stratta RJ, Gaber AO, Shokouh-Amiri MH et al: A prospective comparison of systemic-bladder versus portal-enteric drainage in vascularized pancreas transplantation. Surgery, 2000; $127: 217-26$

26. Fridell JA, Shah A, Milgrom ML et al: Ipsilateral placement of simultaneous pancreas and kidney allografts. Transplantation, 2004; 78: 1074-76 